\title{
Halo and Through-Focus Performance of Four Diffractive Multifocal Intraocular Lenses
}

\author{
Fidel Vega,${ }^{1}$ Francisco Alba-Bueno, ${ }^{1}$ María S. Millán, ${ }^{1}$ Consuelo Varón, ${ }^{1}$ Miguel A. Gil,${ }^{2}$ \\ and Jose A. Buil ${ }^{2}$ \\ ${ }^{1}$ Department d'Òptica i Optometria, Universitat Politècnica de Catalunya, BarcelonaTECH, Terrassa, Spain \\ ${ }^{2}$ Hospital de la Santa Creu i Sant Pau, Barcelona, Spain
}

Correspondence: Fidel Vega, Department d'Optica i Optometria, Universitat Politècnica de Catalunya Violinista Vellsolà 37, 08222 Terrassa, Spain;

fvega@oo.upc.edu.

Submitted: February 3, 2015

Accepted: May 5, 2015

Citation: Vega F, Alba-Bueno F, Millán MS, Varón C, Gil MA, Buil JA. Halo and through-focus performance of four diffractive multifocal intraocular lenses. Invest Ophthalmol Vis Sci. 2015;56:XXX-XXX. DOI:10.1167/ iovs. $15-16600$
Purpose. To compare, as a function of pupil size, the through-focus performance and halo features of four diffractive intraocular lenses (IOLs).

Methods. Three diffractive bifocal IOLs (ReSTOR +2.5 D SV25T0, Tecnis +2.75 D ZKB00, and AT LISA $+3.75 \mathrm{D} 809 \mathrm{M}$ ) and a diffractive trifocal IOL (AT LISA tri $+3.33 \mathrm{D},+1.66 \mathrm{D}$ 839MP) were tested in vitro in a modified International Organization for Standardization eye model. The modulation transfer function (MTF) at the IOLs' foci was obtained with pupils ranging from 2.0 to $5.0 \mathrm{~mm}$. Through-focus MTF curves (at 50 cycles $/ \mathrm{mm}$ ) were compared among all the IOLs. The halo formation and characteristics were obtained from image analysis.

REsults. The multifocal IOLs studied in this work showed, at their foci, secondary out-of-focus images, which originate halos and whose characteristics depend on the lens design and pupil size. The smallest halo occurred for the distance focus of the SV25T0. The distance and near foci of the SV25T0 yielded, respectively, the best and lowest optical quality among the studied IOLs. The distance focus of the ZKB00, AT LISA, and AT LISA tri were of similar quality, but the near focus of the ZKBOO outperformed the near foci of the rest of the IOLs. The IOLs' optical performance gradually deteriorates as pupil increases.

Conclusions. Differences in the design of the diffractive IOLs translate into differences in optical quality at their foci, through-focus performance, and halo features, which can offer further information to surgeons when selecting which IOL to implant.

Keywords: cataract surgery, diffractive bifocal intraocular lens, diffractive trifocal intraocular lens, halo, through-focus performance
$\mathrm{T}$ he development of multifocal intraocular lenses (MIOLs) ${ }^{1}$ offers solutions to satisfy patient demand for spectacle independence after cataract surgery or clear lens extraction. Diffractive MIOLs have shown better optical performance than either of their counterpart refractive MIOLs or accommodating IOLs. $^{2,3}$ In clinical tests, patients implanted with diffractive bifocal IOLs achieved good distance and near visual acuities ${ }^{4,5}$ while showing a significant reduction in visual acuity at intermediate distances. ${ }^{6,7}$ This fact proves that patients have difficulties when performing tasks that require, for instance, computer use, which is important in modern society. To overcome this issue, new designs based on MIOLs with extended depth of focus ${ }^{8}$ or trifocal diffractive MIOLs ${ }^{9,10}$ have been recently proposed, and the first studies on the visual outcomes obtained after implantation of these new trifocal MIOLs are now available. ${ }^{11-13}$

Since bifocal and trifocal diffractive MIOLs are based on the simultaneous image forming principle, they have the drawback that a focused image due to one of the foci will always be overlaid by one (in the case of the bifocal MIOL) or two (in the case of the trifocal MIOL) secondary out-of-focus images originated by the other foci of the MIOL. This effect unavoidably leads to a reduction of the image contrast in comparison to what occurs in patients implanted with monofocal IOLs ${ }^{14}$ and is likely to be among the causes that produce other disturbing optical artifacts such as glare and/or halos. In particular, the perception

Copyright 2015 The Association for Research in Vision and Ophthalmology, Inc. iovs.arvojournals.org | ISSN: 1552-5783 of halos by patients with MIOLs is commonly described when observing distance intense light sources against a dark background, as in the case of night driving. ${ }^{15}$

Optical bench testing of MIOLs ${ }^{3,16}$ is complementary to clinical assessment because, in addition to being objective and patient independent, it has the ability to control factors that are difficult to address in clinical essays such as pupil size, lens alignment, and level of wavefront aberration upon the MIOL. Several studies to date have evaluated diffractive bifocal and trifocal MIOLs in different optical bench configurations to determine through-focus image quality and modulation transfer function (MTF), ${ }^{17,18}$ the light distribution and efficiency of the foci, ${ }^{19,20}$ and the influence of pupil size. ${ }^{21,22}$ However, the formation of the out-of-focus images (superimposed on-axis to the focused one $)^{23,24}$ and their characteristics ${ }^{25}$ in relation to both the diffractive MIOL design and pupil size have been scarcely addressed in the literature. ${ }^{26}$

For a determined pupil size, a diffractive MIOL should provide the best possible optical quality at its focal points and, in addition to this, the largest depth of focus and minimal disturbing side effects like blur or defocus. However, these features are not completely independent; thus, if a particular design of a diffractive lens is intended to optimize one of these factors it should be checked that this does not have a deleterious influence on the other factors. ${ }^{27}$ Hence, it would be interesting to have a more comprehensive description of the 
performance of the MIOLs; thus, the aim in this paper is to study and compare, in an optical bench, the characteristics of the secondary out-of-focus images (which show halo appearance and will be referred to from now on as halo), the influence of the pupil size, and the through-focus performance of four new diffractive MIOLs: AcrySof ReSTOR SV25T0, Tecnis ZKB00, AT LISA 809M, and AT LISA tri 839MP. In addition, a well-known and widely reported monofocal IOL (AcrySof SN60WF) was also tested.

\section{MeTHODS}

\section{MIOL Characteristics}

The AcrySof ReSTOR SV25T0 (Alcon Laboratories, Fort Worth, TX, USA) with +2.5 diopter (D) add power is a diffractive bifocal IOL with an anterior apodized diffractive surface (3.4$\mathrm{mm}$ diameter) within which there is a central refractive zone (1.0-mm diameter approximately). The outer region of the lens to the 6-mm edge is also refractive. The diffractive area presents seven concentric rings with step boundaries of decreasing height, which allows for quite an asymmetric and pupil-dependent light distribution between the distance and near foci. The central and outer refractive parts of the lens are intended for distance vision. The anterior surface of the SV25T0 is designed with negative spherical aberration (SA) of $-0.20 \mu \mathrm{m}$ (value of the $\mathrm{c}[4,0]$ Zernike coefficient for a $6-\mathrm{mm}$ eye pupil) to compensate for the positive SA of the cornea. ${ }^{28,29}$

The Tecnis ZKB00 (AMO Groningen, Groningen, The Netherlands) with $+2.75 \mathrm{D}$ add power is a diffractive bifocal IOL with an anterior aspheric surface and a posterior one with a diffractive profile that covers the full aperture of the lens. This comprises 15 diffractive rings with step boundaries of the same height intended for approximately 50/50 light distribution between the distance and near foci independently of the pupil size. The wavefront-designed aspheric optics of this MIOL produces a maximum SA of $-0.27 \mu \mathrm{m}$ for a $6.0-\mathrm{mm}$ eye pupil.

The AT LISA 809M (Carl Zeiss Meditec AG, Jena, Germany) with $+3.75 \mathrm{D}$ add power is a diffractive bifocal IOL with a design that comprises 29 diffractive rings that fully cover its 6.0-mm-diameter aperture. This design is intended for having a pupil-independent asymmetric light distribution between distance and near foci of approximately 65/35.

The AT LISA tri 839MP (Carl Zeiss Meditec AG) with +3.33 and $+1.66 \mathrm{D}$ add powers for the near and the intermediate foci, respectively, is a trifocal MIOL with the diffractive profile located in the anterior surface. The profile is formed by a trifocal region with a diameter of $4.34 \mathrm{~mm}$ while the outer part of the lens to the 6-mm edge is a bifocal region; that is, it sends light to the far and near foci exclusively. In addition to this, the AT LISA tri has an aspheric design that introduces SA of -0.18 $\mu \mathrm{m}$ for a $6.0-\mathrm{mm}$ eye pupil. ${ }^{10}$

Finally, the monofocal AcrySof SN60WF (Alcon Laboratories), with aspheric optics that induce SA of $-0.20 \mu \mathrm{m}$, was included in our study as a reference for comparison.

All the studied lenses had a base optical power of $20 \mathrm{D}$, which in the case of the MIOLs corresponded to the distance focus.

\section{Experimental Setup for Optical Imaging Quality Assessment}

Assessment of the optical imaging quality of the IOLs was made using an optical test bench with a model eye (artificial cornea plus wet cell) that has been described in detail elsewhere. ${ }^{19,20}$ The setup was in agreement with International Organization

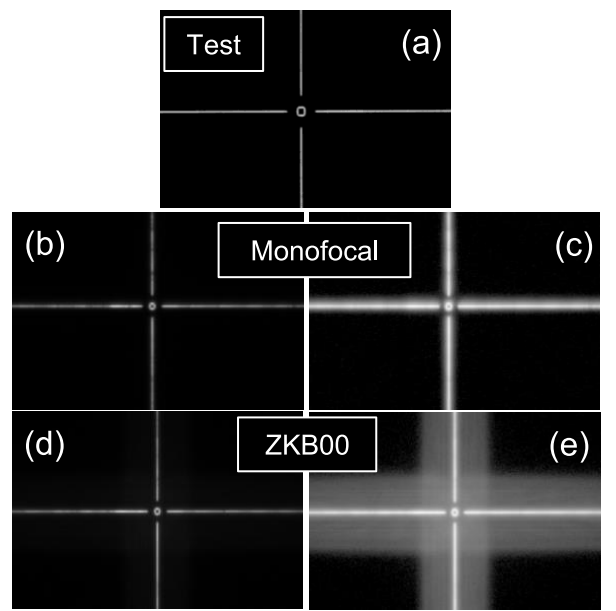

Figure 1. (a) The slit pattern test used as an object for halo and MTF assessment. Images for MTF measurements obtained with monofocal (b) and multifocal (distance focus) (d) IOLs. (c, e) Same images as (b) and (c) but displayed in a logarithmic scale of intensity for halo assessment.

for Standardization (ISO) 11979-2 and 11979-9 ${ }^{30,31}$ recommendations except for the artificial cornea that was not an aberration-free doublet because this was not representative of the average human cornea, which has intrinsic positive SA. ${ }^{28,29}$ Instead, we used a double convex lens that provided a level of SA at the IOL plane of $+0.27 \mu \mathrm{m}$ for a $6.0-\mathrm{mm}$ pupil, similar to the one induced by the human cornea on average. $^{32,33}$ Moreover, it showed a dependence of SA on pupil size close to that of physiological eye models, namely, the Liou-Brenan and Holladay models. ${ }^{19,20}$

The light source was a green light-emitting diode (LED) (LED 525E; Thorlabs, City, State, Country) with emission centered at $525 \mathrm{~nm}$ and with a full-width half-maximum spectral bandwidth of $\pm 15 \mathrm{~nm}$, which illuminated the test object located at the front focal plane of a collimator (200-mm focal length). The test objects were either the USAF 1951, for qualitative image quality assessment, or a four-slit pattern, referred to from now on as slit pattern (Fig. 1a) for MTF measurement and halo characterization. The collimated beam illuminated the model eye with the IOL under test, and an iris diaphragm allows us to have a pupil diameter ranging from 2.0 to $5.0 \mathrm{~mm}$. All the pupil diameters mentioned in this work are referred to the IOL plane. ${ }^{19,20}$

Inserted in the wet cell of the model eye, the diffractive bifocal or trifocal MIOLs simultaneously formed two (distance and near) or three (distance, intermediate, and near) images of the test object. These images were selected and magnified onto an 8-bit charge-coupled device (CCD) camera (Company, City, State, Country) by means of a $\times 10$ infinite corrected microscope (Company, City, State, Country) mounted on a high-precision translation holder. The image recording system (microscope and camera) is diffraction limited with a cutoff frequency at 675 cycles/mm. In addition, the image space of the MIOLs was scanned with the microscope to collect images of the test object at different axial positions between the foci for through-focus analysis. A similar procedure was carried out in the case of the monofocal IOL.

\section{Halo Characterization and MTF Measurement by Image Analysis}

The method to study the formation of the halo and its characteristics using image analysis is illustrated in Figure 1, 
where the best-focused images of the slit pattern formed by the distance focus of one of the MIOLs under study and the monofocal IOL are shown. To improve the signal-to-noise ratio, each image is the result of averaging eight image frames. Since the images formed by the MIOLs contain the superposition of a bright focused image of the slit pattern along with one or several dimmer out-of-focus images, a large dynamic range is required for data presentation. In such cases and when image display or printing is involved, it is common practice to use logarithmic scale. For instance, while it is difficult to notice the differences between the images of Figures $1 \mathrm{~b}$ and $1 \mathrm{~d}$, which are displayed at linear scale, they become clearly distinguishable in logarithmic scale (Figs. 1c, 1e). The reason is that the logarithmic function compresses the dynamic range of an image by simultaneously expanding the values of low-intensity pixels and compressing higher-level pixels, ${ }^{34}$ and then it is possible to visualize relatively weak backgrounds in the presence of intense signals.

The aforementioned change into a logarithmic scale enables one to illustrate the differences between the images formed by the monofocal IOL and those formed by the MIOLs and makes possible the assessment of the formation (or not) of the halo (compare Figs. 1c, 1d) and some of its features, such as width and shape, that will eventually influence the imaging quality of the lenses as reported in earlier work. ${ }^{23,26}$

Among the different image quality metrics, the MTF has been used extensively for image quality evaluation of MIOLs ${ }^{3,18,21,22}$; and recently, Felipe et al..$^{35}$ found a significant correlation between visual acuity of patients and average MTF of the MIOLs they were implanted with.

In this study the MTF was obtained as reported by Simpson $^{23}$ from the Fourier transform of the line spread function of the images of the slit pattern (Fig. 1). The criterion for determining the best focus planes was to choose those that maximized the area under the MTF curve. The MTF curves were obtained for pupil sizes ranging from 2.0 to $5.0 \mathrm{~mm}$. Through-focus MTF at the spatial frequency of 50 cycles $/ \mathrm{mm}$, as recommended in ISO 11979-2 and 11979-9, was obtained for $3.0-$ and $4.5-\mathrm{mm}$ pupils. The axial scanning in the MIOL image space for the through-focus analysis was made from +1.75 to $-5.0 \mathrm{D}$ in $0.15 \mathrm{D}$ steps.

\section{Results}

We have studied the formation and characteristics of the halo by considering the distance focus of the MIOLs. Figure 2 shows the images of the slit pattern obtained with the MIOLs of this study and displayed at logarithmic scale of intensity. Supplementary Figure S1 shows the same images but in linear scale of intensity. The images obtained with the monofocal IOL are also included at the top of these figures. From Figure 2 it is evident that, in comparison with the monofocal IOL, all the MIOLs have a wide halo in the background surrounding the focused image of the slit pattern. As for each MIOL, with the exception of the SV25T0, the halo size increases as the pupil increases from 3.0 to $4.5 \mathrm{~mm}$. Finally, and for both pupil sizes, the largest halos are obtained with the bifocal and trifocal AT LISAs, which are also the MIOLs with highest add powers for the near focus $(+3.75$ and $+3.33 \mathrm{D}$, respectively).

Figure 3 shows, for a pupil size of $3.0 \mathrm{~mm}$, the experimental images of the USAF test obtained in the distance and near foci of the bifocal MIOLs and in an intermediate plane right in the middle between the two foci, as well as the images obtained in the distance, intermediate, and near foci of the trifocal MIOL. The AT LISA tri is the only MIOL that shows a true intermediate focus where it is possible to resolve more groups of the USAF test, even though notoriously blurred. Furthermore, the

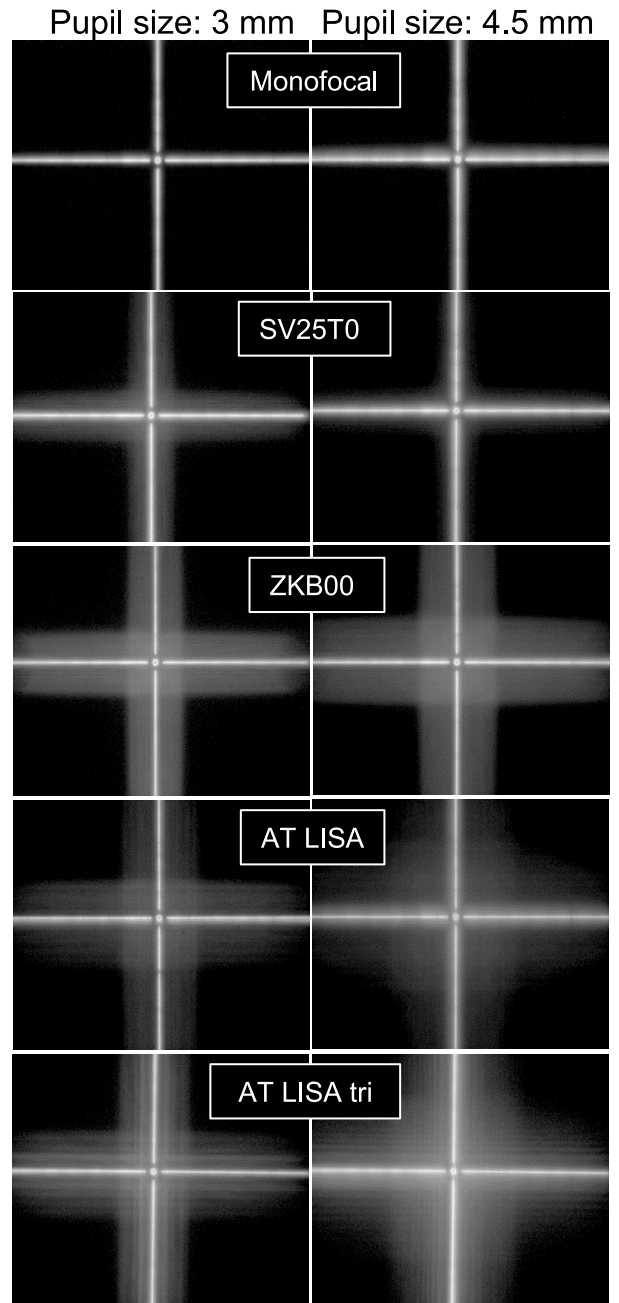

Figure 2. Images of the slit pattern obtained with pupils of 3.0 and $4.5 \mathrm{~mm}$. The images correspond to the focus of the monofocal IOL $(t o p)$ and distance focus of the MIOLs. All images were recorded in the same experimental conditions of exposure time and LED power and are shown in a logarithmic scale of intensity to enable halo visualization.

imaging quality of the bifocal MIOLs at intermediate planes appears to be quite low in comparison with their distance and near foci.

Some of the above-mentioned characteristics can also be observed in the images of the slit pattern shown in Figure 4. In addition, this figure shows that the presence of halos is not restricted to the distance focus but is clearly observed in every other focus of the MIOLs.

The MTFs of the MIOLs, obtained from the images of the slit pattern with 3.0- and 4.5-mm pupils, are shown in Figure 5. For the sake of comparison, the MTF corresponding to the monofocal IOL, which must be compared to the ones computed for the distance foci of the MIOLs, is included in these plots. For both pupil sizes, the MTF of the monofocal IOL reaches higher values than any other MTF corresponding to the distance focus of the MIOLs. However, in the case of the SV25T0 with a 4.5-mm pupil, both MTFs are fairly close.

For a pupil size of $3.0 \mathrm{~mm}$, and comparing the distance foci of the MIOLs, the best MTF corresponds to the bifocal SV25T0. Conversely, this MIOL is the one with the worst MTF in the case of the near foci. The bifocal ZKB00 shows similar MTF curves, and hence similar optical imaging quality, for its 


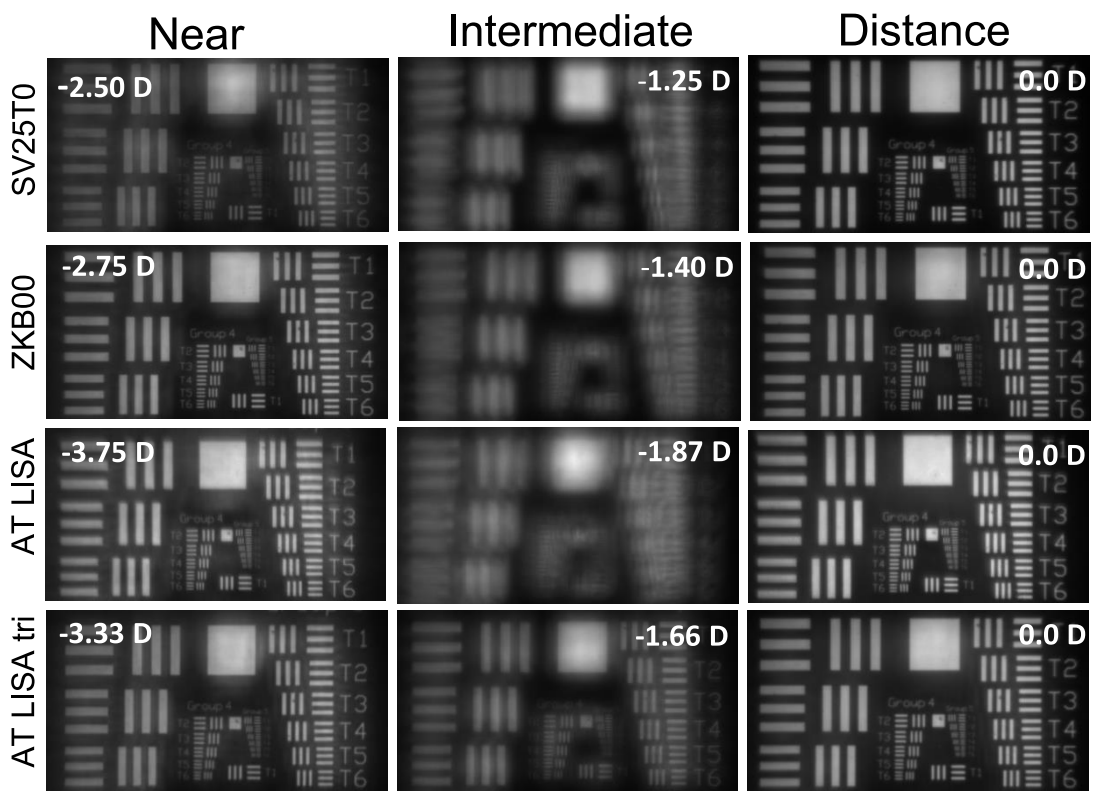

FIGURE 3. Images of the USAF test obtained with a 3.0-mm pupil, at the foci and at an intermediate plane of the bifocal MIOLs and at the three foci of the trifocal MIOL. The position of the planes, in diopters, is referred to the distance focus of each MIOL.

distance and near foci. The same behavior is observed in the case of the bifocal AT LISA. The trifocal AT LISA, on the other hand, has an intermediate focus that outperforms the intermediate planes of all the bifocal MIOLs. Indeed, and as expected from the reduced quality of the images at intermediate planes (Figs. 5, 6), the MTF curves of the bifocal MIOLs at these planes drop to zero very quickly, thus confirming a low optical imaging quality. Similar results were obtained with the 4.5-mm pupil, although in this case the MTFs are slightly worse.

To get further insight concerning the influence of the pupil size on the optical performance of the MIOLs, we obtained the MTF curves at the foci for pupils ranging from 2.0 to $5.0 \mathrm{~mm}$ and compared the values among the MIOLs at the spatial frequency of 50 cycles $/ \mathrm{mm} .{ }^{21,22}$ In our setup, this frequency approximately corresponds to an angular spatial frequency of $30 \mathrm{cyc} / \mathrm{deg}$. The results, plotted in Figure 6, confirm as a general trend that the MTF and thus the optical imaging quality of the MIOLs tend to decrease as the pupil increases, with the bifocal ZKB00 showing the minimum dependence on the pupil size. Moreover, these results further confirm that the distance focus of the SV25T0 MIOL is the one with the best optical performance among the multifocal IOLs studied; but at the same time, this lens has the near focus with the lowest optical imaging quality for all pupil sizes.

Figure 7 shows the through-focus MTF curves, evaluated at the spatial frequency of $50 \mathrm{cycles} / \mathrm{mm}$, for the four diffractive MIOLs with a 3.0-mm pupil. The position of the MTF peaks is related to the main foci of the MIOLs; hence these curves emphasize the different performance and optical imaging quality of the foci of the MIOLs as a function of their particular design. The highest MTF value for the distance focus (0.0 D) corresponds to the bifocal SV25T0 with a score of $\approx 0.40$, whereas the other three MIOLs (ZKB00, AT LISA, and AT LISA tri) have values in the range of 0.25 to 0.28 . For comparison, the MTF of the monofocal SN60WF (not shown in Fig. 7) reached a score of 0.65 in these conditions, which is in good agreement with the result reported in an earlier work. ${ }^{18}$ The ZKBOO is the only MIOL that has similar MTF peaks at distance and near foci (located at $-2.75 \mathrm{D}$ ), and the values measured are in good agreement with those provided by the manufacturer in the technical datasheet. Interestingly, the MTF of the ZKBOO at an intermediate plane of $-2.50 \mathrm{D}$ is higher than that of the SV25T0, even though the latter is specifically designed to have its near foci at $-2.50 \mathrm{D}$. Another interesting comparison can be made between the AT LISA (bifocal) and the AT LISA tri because the MTF peaks and hence the optical imaging quality of the distance $(0.0 \mathrm{D})$ and near foci $(-3.75$ and $-3.33 \mathrm{D}$, respectively) of these two lenses are very similar, but the trifocal has the advantage of showing an additional intermediate peak (at $-1.66 \mathrm{D})$. The MTF score of this intermediate peak $(0.12)$ is similar to the one found for the near focus of the SV25T0.

\section{Discussion}

It is worth emphasizing that in an optical test bench, what can be assessed is the formation and characteristics of the secondary out-of-focus images produced by the MIOLs, which gives rise to a background that shows halo appearance and blurs the focused image. The presence of such background is a necessary but not sufficient condition for the perception of the halo by a patient because even though Stevens' power law predicts a logarithmic behavior of the sensory magnitude or perception, ${ }^{36}$ it is not possible to infer from it that the figures displayed at logarithmic scale are a direct representation of what a patient would see.

Our results (Fig. 4) show that the images obtained in each focus plane of the MIOLs have a background or halo that originates blur and causes reduction of the image contrast. Moreover, these results allow for a comparison between the halo produced by MIOLs at their distance focus and the blur produced by a monofocal IOL, which is mainly due to aberrations and is considerably weaker (Fig. 2). In the case of the MIOLs, the halo is primarily due to the intensity and size of the out-of-focus image (or images) corresponding to the other foci of the lens, which turns out to depend on both the add power ${ }^{17,26}$ and how the MIOL distributes the energy among the foci. ${ }^{19,37}$ For a given pupil size, the fraction of the energy that is out of focus (and thus the halo) also depends in a complex way on a variety of factors such as the energy 

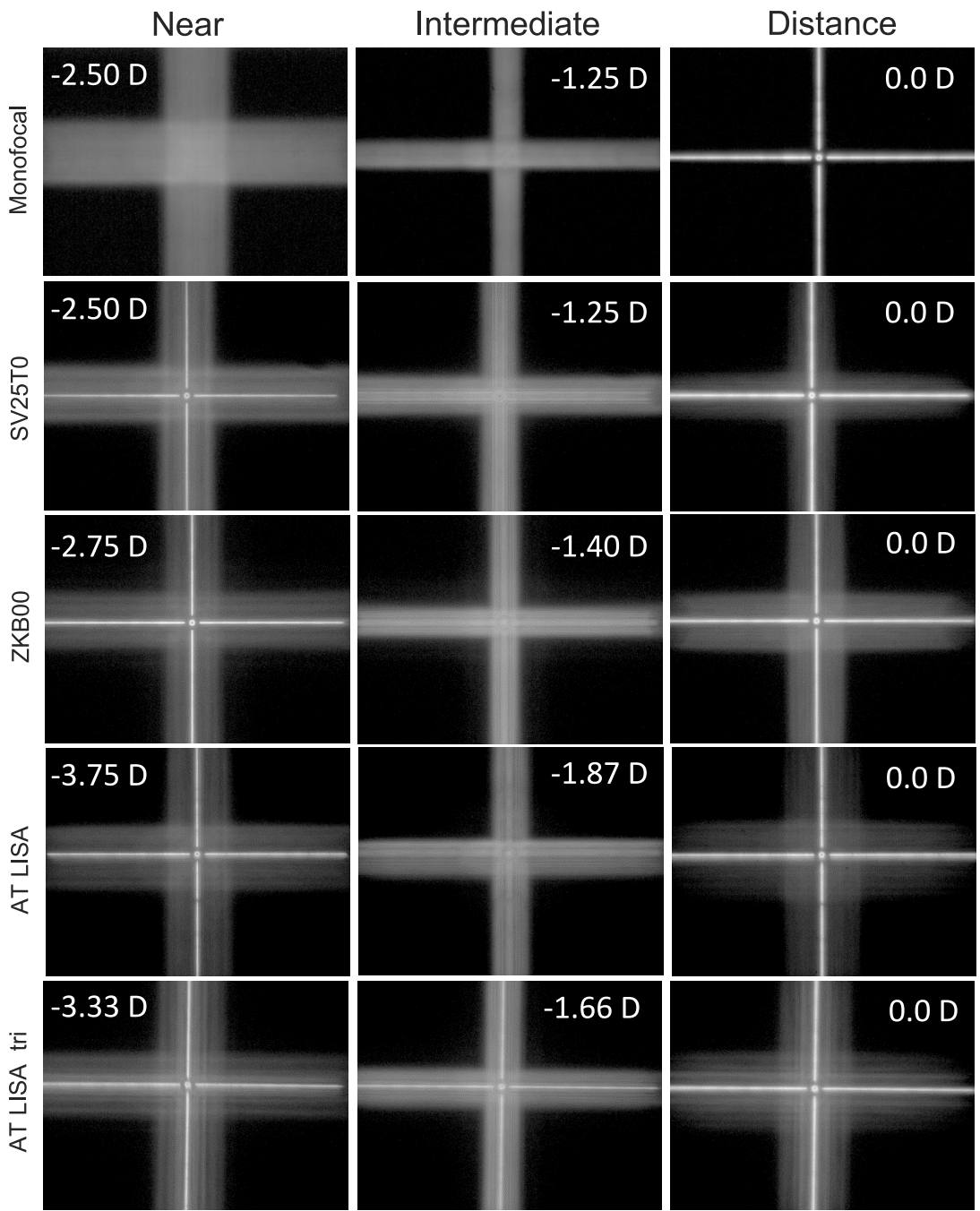

Figure 4. Images of the slit pattern obtained with a 3.0-mm pupil, at the foci and at an intermediate plane of the bifocal MIOLs and at the three foci of the trifocal MIOL. The position of the planes, in diopters, is referred to the distance focus of each MIOL. All the images are shown in a logarithmic scale of intensity.

expended in higher diffraction orders ${ }^{37}$ scattering produced by the diffractive steps, ${ }^{38}$ and the residual level of aberrations, mainly SA, once the MIOL has compensated (totally or partially) the positive SA of the cornea. ${ }^{20,33}$

As for the optical quality of the MIOLs, their different designs are intended to obtain the best possible images at their focal points; hence, the comparison of the optical quality of the lenses has been carried out at their best foci. Otherwise, and especially in the case of the near focus (we recall that all the studied MIOLs had different add powers), the use of a fixed plane at a particular vergence could benefit the IOL whose add power approached the selected vergence the most.

In the case of the distance focus, this study shows that the SV25T0 reaches the highest MTF score and thus the best optical imaging quality, which is in agreement with previous work. ${ }^{39}$ This is consistent with the design of this lens: a low add power and an apodized diffractive profile to enhance the performance of its distance focus. For instance, for a 3-mm pupil, the SV25T0 MIOL has the smallest number of diffractive steps, a small remnant level of SA (once the lens has partially compensated for the SA of the cornea), and the lowest diffraction efficiency for the near focus (only $20 \%$, whereas the ZKB00, AT LISA, and AT LISA tri have diffraction efficiencies of $48 \%, 35 \%$, and $30 \%$, respectively). ${ }^{10,40}$ Consequently, the
SV25T0 is the MIOL where the factors (diffraction efficiency of the near focus, scattering produced by the diffractive steps, and remnant SA) that determine the amount of out-of-focus energy (or halo) at the distance focus and contribute to degrade its optical quality have the smallest contribution. Conversely, the SV25T0 has the lowest optical quality at the near focus. Altogether, these results are consistent with recent clinical findings showing that patients implanted with the SV25T0 had excellent distance vision (comparable to that of patients with monofocal IOL) but their near vision was worse than that of patients with other types of MIOLs. ${ }^{40}$

The other MIOLs (ZKB00, AT LISA, and AT LISA tri) show a more balanced performance in their foci, with their near focus outperforming the near focus of the SV25T0 as deduced from the MTF values for all pupil size conditions (Fig. 6). Since it has been suggested that the imaging quality of the MIOLs (determined by the MTF) could have an influence on the visual quality of patients, ${ }^{35,41}$ and also because these MIOLs have higher add powers, one could infer that they could be a good choice for patients with demanding near vision activities. However, according to our results (Fig. 2), these lenses are also prone to induce visual disturbances associated with halos. ${ }^{26}$ This drawback must be carefully considered with patients 


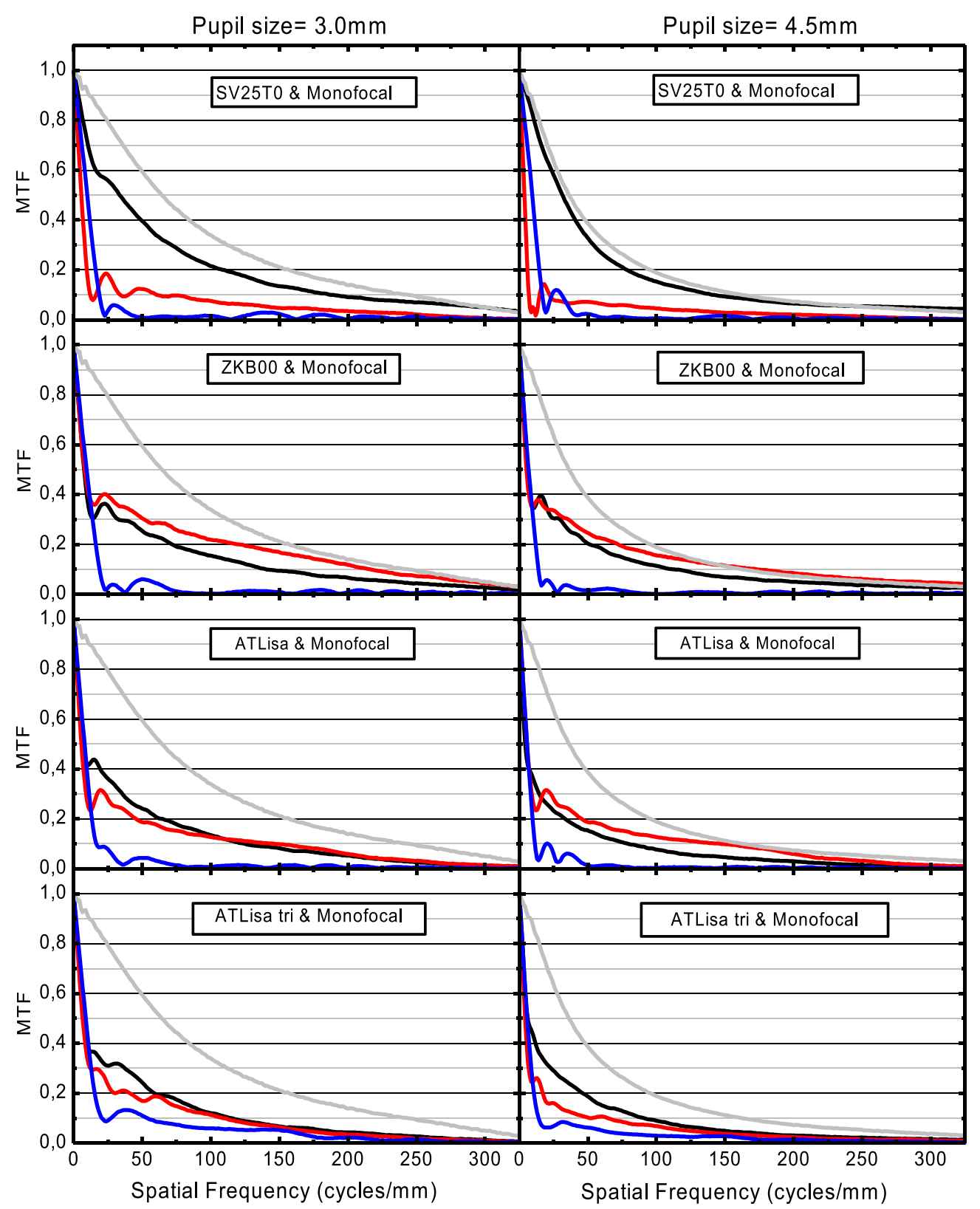

Figure 5. MTF curves of the monofocal IOL (gray line) and the MIOLs obtained at their respective distance (black line), near (red line), and intermediate (blue line) planes with 3.0- and 4.5-mm pupils.

whose expectations after surgery were a low level of visual disturbance. ${ }^{40}$

The case of the AT LISA tri deserves further explanation. The MTF values of this MIOL as a function of the pupil size (Fig. 6) and the through-focus MTF (Fig. 7) are in quite good agreement with earlier reported works. ${ }^{22,39}$ The AT LISA tri has MTF scores for its distance and near foci similar to those of the bifocal AT LISA, but the trifocal MIOL is the only one that provides a real intermediate focus. This result suggests that the trifocal lens may provide better intermediate vision without compromising near and distance vision. Interestingly, while conventional defocus curves obtained in patients with bifocal IOLs show two peaks corresponding to distance and near vision with a significant drop in visual acuity at intermediate distance, ${ }^{42,43}$ the first outcomes with the AT LISA tri show a comparative smaller reduction of intermediate visual acuity with no significant differences in the range from -2.0 to $-1.0 \mathrm{D}$ (at the spectacle plane). ${ }^{13,44}$ However, we have also shown (Supplementary Fig. S1) that the AT LISA tri would more likely produce the largest halo in the distance focus for a $4.5-\mathrm{mm}$ pupil. These two findings may help to explain the results of recent clinical studies with this MIOL showing that intermediate vision of patients efficiently improved, but at the same time, $10 \%$ of patients reported perception of halos. ${ }^{13}$

As additional comments, we recall that our results were obtained from on-axis analyses, that is, with the MIOLs aligned with the optical system. Earlier work ${ }^{45,46}$ has shown that tilt and/or decentration of IOLs have an impact on their optical performance. In addition, the human eye naturally includes pupil decentration (with respect to the cornea and crystalline lens) as well as lens tilt. Another potential issue of the study concerns the SA of the artificial cornea. Although there is a general consensus about the need to use artificial cornea models with positive SA to properly test IOLs of aspheric 

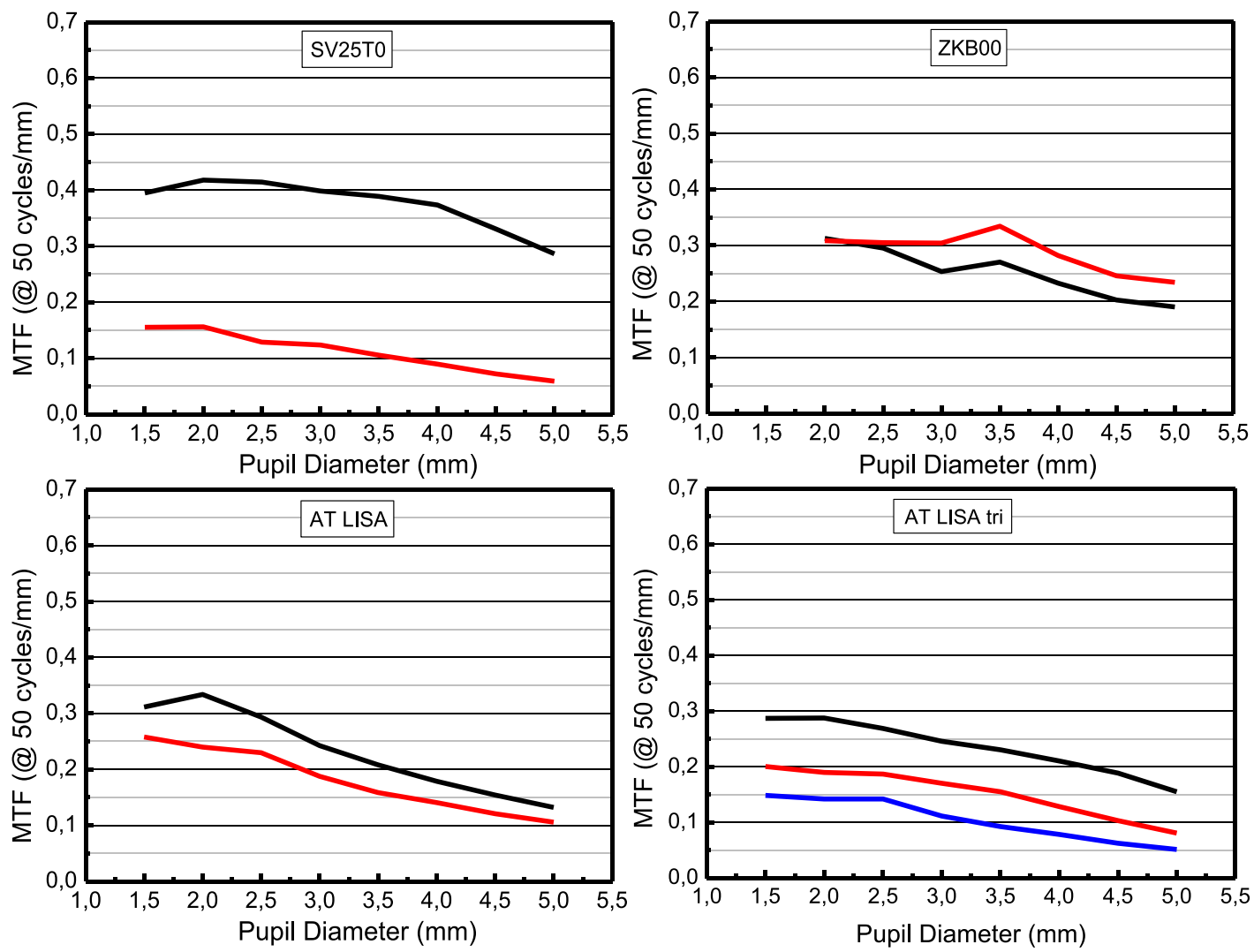

Figure 6. MTF values at 50 cycles/mm as a function of the pupil size for the distance (black line), near (red line), and intermediate (blue line) foci of the MIOLs.

design, ${ }^{33}$ there is not yet agreement about the specific value of the corneal SA that should be used. For instance, Pieh and coworkers $^{45}$ used three corneas with SA $(6-\mathrm{mm}$ pupil) of $+0.054,+0.172$, and $+0.416 \mu \mathrm{m}$, respectively, for in vitro testing of monofocal IOLs. The model eye cornea of Carson et al. ${ }^{47}$ had a SA of $+0.2 \mu \mathrm{m}$ and was used to test the MIOLs SV25T0 and ATLISA tri. The artificial cornea used in our eye model was designed with a SA of $+0.27 \mu \mathrm{m}$ for a 6-mm pupil. Taking into account the SA of the MIOLs (SV25T0: $-0.20 \mu \mathrm{m}$; ZKB00: $-0.27 \mu \mathrm{m}$; AT LISA bi and AT LISA tri: $-0.18 \mu \mathrm{m}$ ), the maximum remnant SA would be only $+0.07 \mu \mathrm{m}$ in the case of the SV25T0 and $+0.09 \mu \mathrm{m}$ for the AT LISA bifocal and trifocal.

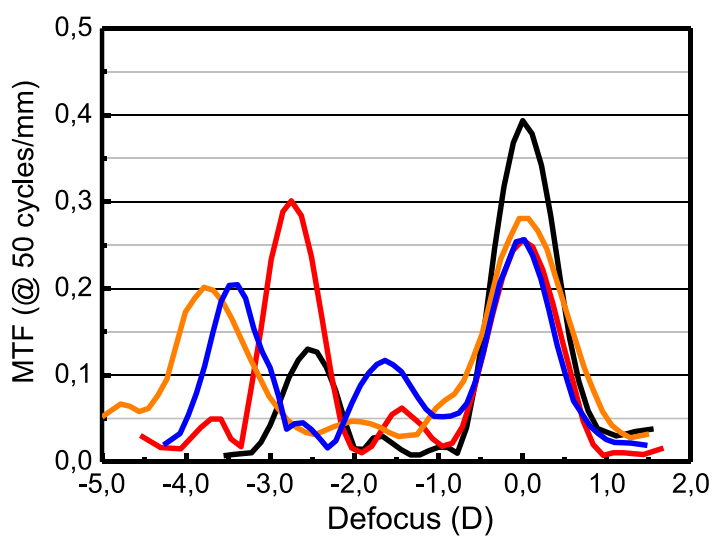

Figure 7. Through-focus MTF curves for the SV25T0 (black line), ZKBOO (red line), AT LISA (orange line), and AT LISA tri (blue line). The pupil size is $3.0 \mathrm{~mm}$ and the abscissa axis has the origin $(0.0 \mathrm{D}$ defocus) at the distance focus of each lens.
Since the maximum pupil diameter used in this work was 4.5 $\mathrm{mm}$, one expects even smaller remnant values of SA, ${ }^{48}$ and hence differences in the optical performance of the MIOLs associated with differences in the SA compensation can be neglected. Finally, the measurements of the MIOLs were performed using a single wavelength of $525 \mathrm{~nm}$ close to the maximum peak sensitivity of the human eye in photopic conditions. However, the MIOLs will commonly work under polychromatic light such as daylight. All these issues should be taken into account with respect to a closer approximation to patients implanted with this type of MIOL.

\section{Acknowledgments}

Supported by project DPI2013-43220-R from the Spanish Ministerio de Economía y Competitividad y Fondos FEDER.

Disclosure: F. Vega, None; F. Alba-Bueno, None; M.S. Millán, None; C. Varón, None; M.A. Gil, None; J.A. Buil, None

\section{References}

1. de Vries N, Nuijts R. Multifocal intraocular lenses in cataract surgery: literature review of benefits and side effects. $J$ Cataract Refract Surg. 2013;39:268-278.

2. Artigas JM, Menezo JL, Peris C, Felipe A, Díaz-Llopis M. Image quality with multifocal intraocular lenses and the effect of pupil size: comparison of refractive and hybrid refractivediffractive designs. J Cataract Refract Surg. 2007;33:21112117.

3. Maxwell WA, Lane SS, Zhou F. Performance of presbyopia correcting intraocular lenses in distance optical bench tests. $J$ Cataract Refract Surg. 2009;35:166-171. 
4. Alió JL, Elkady B, Ortiz D, Bernabeu G. Clinical outcomes and intraocular optical quality of a diffractive multifocal intraocular lens with asymmetrical light distribution. J Cataract Refract Surg. 2008;34:942-948.

5. Blaylock JF, Si Z, Vickers C. Visual and refractive status at different focal distances after implantation of the ReSTOR multifocal intraocular lens. J Cataract Refract Surg. 2006;32: 1464-1473.

6. Alfonso JC, Fernández-Vega L, Begoña BM, Montés-Micó R. Prospective visual evaluation of apodized diffractive intraocular lenses. J Cataract Refract Surg. 2007;33:1235-1243.

7. Petermeier K, Szurman P. Subjective and objective outcome following implantation of the apodized diffractive AcrySof ReSTOR. Ophthalmology. 2007;104:406-408.

8. Fernández D, Barbero S, Dorronsoro C, Marcos S. Multifocal intraocular lens providing optimized through-focus performance. Optics Lett. 2013;38:5303-5306.

9. Gatinel D, Pagnoulle C, Houbrechts Y, Gobin L. Design and qualification of a diffractive trifocal optical profile for intraocular lenses. J Cataract Refract Surg. 2011;37:20602067.

10. Montin JF. Achieving spectacle independence with the ATLISA tri 839MP. Cataract \& Refractive Surgery Today Europe. 2012;3(March suppl):16-19.

11. Cochener B, Vryghem J, Rozot P, et al. Visual and refractive outcomes after implantation of a fully diffractive trifocal lens. Clin Ophthalmol. 2012;6:1421-1427.

12. Sheppard AL, Shah S, Bhatt U, Bhogal G, Wolffsohn JS. Visual outcomes and subjective experience after bilateral implantation of a new diffractive trifocal intraocular lens. J Cataract Refract Surg. 2013;39:343-349.

13. Mojzis P, Peña-García P, Liehneova I, Ziak P, Alió J. Outcomes of a new diffractive trifocal intraocular lens. $J$ Cataract Refract Surg. 2014;40:60-69.

14. Gil MA, Varón C, Cardona G, Vega F, Buil J. Comparison of far and near contrast sensitivity in patients symmetrically implanted with multifocal and monofocal IOLs. Eur $J$ Ophthalmol. 2014;24:44-52.

15. Choi J, Schwiegerlin J. Optical performance measurement and night driving simulation of Restor, Rezoom, and Tecnis multifocal intraocular lenses in a model eye. J Refract Surg. 2008;24:218-222.

16. Kim MJ, Zheleznyak L, MacRae S, Tchah H, Yoon G. Objective evaluation of through-focus optical performance of presbyopia-correcting intraocular lenses using an optical bench system. J Cataract Refract Surg. 2011;37:1305-1312.

17. Pepose JS, Wang D, Altmann GE. Comparison of through-focus image sharpness across five presbyopia-correcting intraocular lenses. Am J Ophthalmol. 2012;154:20-28.

18. Gatinel D, Houbrechts Y. Comparison of bifocal and trifocal diffractive and refractive intraocular lenses using an optical bench. J Cataract Refract Surg. 2013;39:1093-1099.

19. Vega F, Alba-Bueno F, Millán MS. Energy distribution between distance and near images in apodized diffractive multifocal intraocular lenses. Invest Ophthalmol Vis Sci. 2011;52:56955710.

20. Vega F, Alba-Bueno F, Millán MS. Energy efficiency of a new trifocal intraocular lens. $J$ Eur Opt Soc Rap Pub. 2014;9: 14002-14008

21. Montés-Micó R, Madrid-Costa D, Ruiz-Alcocer J, Ferrer-Blasco T, Pons AM. In vitro optical quality differences between multifocal apodized diffractive intraocular lenses. $J$ Cataract Refract Surg. 2013;39:928-936.

22. Ruiz-Alcocer J, Madrid-Costa D, García-Lázaro S, Ferrer-Blasco T, Montés-Micó R. Optical performance of two new trifocal intraocular lenses: through-focus MTF and influence of pupil size. Clin Experiment Ophthalmol. 2014;42:271-276.
23. Simpson MJ. Diffractive multifocal intraocular lens image quality. Appl Opt. 1992;31:3621-3626.

24. Pieh S, Lackner B, Hanselmayer G, et al. Halo size under distance and near conditions in refractive multifocal intraocular lenses. Br J Ophthalmol. 2001;85:816-821.

25. Gobbi PG, Fasce F, Bozza S, Calori G, Brancato R. Far and near visual acuity with multifocal intraocular lenses in an optomechanical eye model with imaging capability. $J$ Cataract Refract Surg. 2007;33:1082-1094.

26. Alba-Bueno F, Vega F, Millán MS. Halos and multifocal intraocular lenses: origin and interpretation. Arch Soc Esp Oftalm. 2014. doi:10.1016/j.oftal.2014.01.002.

27. Romero LA, Millán MS, Jaroszewicz Z, Kolodziejczyk A. Double peacock eye optical element for extended focal depth imaging with ophthalmic applications. J Biomed Opt. 2012;17: 046013-046020

28. Guirao A, Redondo M, Artal P. Optical aberrations of the human cornea as a function of age. J Opt Soc Am A Opt Image Sci Vis. 2000;17:1697-1702.

29. Wang L, Dai E, Koch DD, Nathoo A. Optical aberrations of the human anterior cornea. J. Cataract Refract Surg. 2003;29: 1514-1521.

30. International Organization for Standardization (ISO). ISO 11979-2: Ophthalmic implants - intraocular lenses - Part 2: Optical properties and test methods. ISO; 1999.

31. International Organization for Standardization (ISO). ISO 11979-9: Ophthalmic implants - intraocular lenses - Part 9: Multifocal intraocular lenses. ISO; 2006.

32. Vega F, Millán MS, Wells B. Spherical lens versus aspheric artificial cornea for intraocular lens testing. Optics Lett. 2010; 35:1539-1541

33. Norrby S, Piers P, Campbell CH, van der Mooren M. Model eyes for evaluation of intraocular lenses. Appl Opt. 2007;46:65956605.

34. Gonzalez RC, Woods RE. Digital Image Processing. 3rd ed. Upper Saddle River, NJ: Pearson Prentice Hall; 2008;

35. Felipe A, Pastor F, Artigas JM, Diez-Ajenjo A, Gené A, Menezo JL. Correlation between optics quality of multifocal intraocular lenses and visual acuity. J Cataract Refract Surg. 2010;36:557562 .

36. Norton TT, Corliss DA, Bailey JE. The Psychophysical Measurement of Visual Function. Amsterdam: Butterworth Heinemann; 2002;

37. Castignoles F, Flury M, Lepine T. Comparison of the efficiency, MTF and chromatic properties of four diffractive bifocal intraocular lens designs. Opt Express. 2010;18:5245-5256.

38. Meyers MM, Albrecht RE. Technique to eliminate scattered light in diffractive optical elements. U.S. Patent 5,801,889. September 1, 1998.

39. Madrid-Costa D, Ruiz-Alcocer J, Ferrer-Blasco T, García-Lázaro S, Montés-Micó R. Optical quality differences between three multifocal intraocular lenses: bifocal low add, bifocal moderate add and trifocal. $J$ Refract Surg. 2013;29:749-754.

40. Gundersen KG, Potvin R. Comparative visual performance with monofocal and multifocal intraocular lenses. Clin Ophthalmol. 2013;7:1979-1985.

41. Peris-Martínez C, Artigas JM, Sánchez-Cortina I, Felipe A, DíezAjenjo A, Menezo JL. Influence of optical quality on contrast sensitivity and visual acuity in eyes with a rigid or flexible phakic intraocular lens. J Cataract Refract Surg. 2009;35: 1911-1917.

42. de Vries N, Webers CAB, Montés-Micó R, Ferrer-Blasco T, Nuijts RM. Visual outcomes after cataract surgery with implantation of $\mathrm{a}+3.00 \mathrm{D}$ or $+4.00 \mathrm{D}$ aspheric diffractive multifocal intraocular lenses: comparative study. $J$ Cataract Refract Surg. 2010;36:1316-1322. 
43. Packer M, Chu YR, Waltz KL, et al. Evaluation of the aspheric Tecnis multifocal intraocular lens: one-year results from the first cohort of the Food and Drug Administration clinical trial. Am J Opbthalmol. 2010;149:577-584.

44. Marques EF, Ferreira TB. Comparison of visual outcomes of 2 diffractive trifocal intraocular lenses. J Cataract Refract Surg. 2015; $41: 354-363$

45. Pieh S, Fiala W, Malz A, Stork W. In vitro Stehl ratios with spherical, aberration free, average, and customized spherical aberration correcting intraocular lenses. Invest Opbthalmol Vis Sci. 2009;50:1264-1270.
46. Mackelvie J, McArdle B, McGhee C. The influence of tilt, decentration, and pupil size on the higher-order aberration profile of aspheric intraocular lenses. Opbthalmology. 2011; 118:1724-1731.

47. Carson D, Hill WE, Hong X, Karakelle M. Optical bench performance of AcrySof IQ ReSTOR, AT LISA tri, and FineVision intraocular lenses. Clin Opbthalmol. 2014;8: 2105-2113.

48. Eom Y, Yoo E, Kang S, Kim H, Song J. Change in efficiency of aspheric intraocular lenses based on pupil diameter. Am J Ophthalmol. 2013;155:492-498. 
1. Author: This article has been lightly edited for grammar, style, and usage. Please compare against your original document and make changes on these pages. Please limit your corrections to substantive changes that affect meaning. If no change is required in response to a question, please write "OK as set" in the margin. Copy editor

2. Author: Carefully check the spelling and order of the author names and check the affiliations for all of the authors.

3. Author: Make sure that ALL funding/financial support is listed in the information following the Acknowledgments.

4. Author: Please confirm wording of précis (a 1-2 sentence summary or brief description of your paper which will be added to the Table of Contents): Differences in the design of the diffractive IOLs translate into differences in optical quality at their foci, through-focus performance, and halo features, which can offer further information to surgeons when selecting which IOL to implant. Copy editor

5. Author: Short title, "Halo and Through-Focus of Four Diffractive MIOLs," created for running header per journal style. Please confirm wording. Copy editor

6. Author. Sentence starting "The anterior surface": Please confirm that "c[4,0]" is correct. Copy editor

7. Author. Sentence starting "The light source": Please add location information for Thorlabs where placeholders are. Copy editor

8. Author. Paragraph starting "Inserted in": Please insert company information for the camera and microscope. Copy editor

9. Author. Ref. 20: Please check journal abbreviation - could not find on Internet. Copy editor

10. Author. Ref. 22: Revised entry per Internet listing - please confirm. Copy editor

11. Author. Ref. 34: Please insert page numbers if applicable. Copy editor

12. Author. Ref. 36: Please insert page numbers if applicable. Copy editor

13. Author. Figure 1 caption: Should "(c)" be "(d)"? Copy editor

14. Author. Figure 5, 6, and 7 captions: Please see if edits to these captions are correct. Copy editor 